Jean-Yves MOLLIER et Eliana de FREITAS DUTRA [dir.], L'imprimé dans la construction de la vie politique. Brésil, Europe, Amériques, XVIII -XX ${ }^{e}$ siècle

Rennes, Presses universitaires de Rennes, collection « Des Amériques ", 2015

\title{
Sébastien Rozeaux
}

\section{CpenEdition}

Journals

Édition électronique

URL : http://journals.openedition.org/rh19/5130

DOI : $10.4000 /$ rh 19.5130

ISSN : 1777-5329

Éditeur

La Société de 1848

\section{Édition imprimée}

Date de publication : 1 décembre 2016

Pagination : 192-195

ISSN : 1265-1354

\section{Référence électronique}

Sébastien Rozeaux, « Jean-Yves MOLLIER et Eliana de FREITAS DUTRA [dir.], L'imprimé dans la construction de la vie politique. Brésil, Europe, Amériques, XVIIIe-XXe siècle », Revue d'histoire du XIXe siècle [En ligne], 53 | 2016, mis en ligne le 21 février 2017, consulté le 25 septembre 2020. URL : http:// journals.openedition.org/rh19/5130; DOI : https://doi.org/10.4000/rh19.5130 


\section{Jean-Yves MOLLIER et Eliana de FREITAS DUTRA [dir.], L'imprimé dans la construction de la vie politique. Brésil, Europe, Amériques, XVIII ${ }^{e}-X X^{e}$ siècle}

Rennes, Presses universitaires de Rennes, collection « Des Amériques », 2015

\section{Sébastien Rozeaux}

\section{RÉFÉRENCE}

Jean-Yves MOLLIER et Eliana de FREITAS DUTRA [dir.], L'imprimé dans la construction de la vie politique. Brésil, Europe, Amériques, XVIII ${ }^{e}-X X^{e}$ siècle, Rennes, Presses universitaires de Rennes, collection « Des Amériques », 2015, 502 p., $26 €$.

La publication de ces actes fait suite à un colloque organisé par le département d'histoire de l'université fédérale de Minas Gerais, au Brésil, et le Centre d'histoire culturelle des sociétés contemporaines de l'université de Versailles Saint-Quentin-enYvelines, en France. La plupart des 28 articles traitent de la question de l'imprimé dans la vie politique au Brésil, sur le temps long et dans une perspective résolument atlantique. En introduction, Eliana de Freitas Dutra et Jean-Yves Mollier soulignent l'importance de l'imprimé dans les processus historiques de construction nationale et défendent les vertus d'une histoire croisée - politique, sociale et culturelle - de l'imprimé, compte tenu de son « rôle irremplaçable [...] dans la formation et l'évolution des opinions publiques » (p. 14). Ce volume de 500 pages se divise en dix sections : un tel émiettement peine selon nous à rendre pleinement justice à la grande qualité de la plupart des contributions, faute d'en dégager les principales lignes de force. D'où notre 
choix ici de mettre en exergue trois axes problématiques qui illustrent la richesse du renouvellement de l'historiographie au Brésil, notamment sur son versant culturel. Notre attention se concentrera ici sur les articles portant sur le « long XIX ${ }^{e}$ siècle ».

Le principal mérite de cet ouvrage réside sans nul doute dans les nombreuses réflexions portant sur les politiques publiques de l'imprimé dans l'espace impérial portugais. Ainsi, Lilia Moritz Schwarcz étudie l'histoire chaotique de la bibliothèque royale au Portugal ; bibliothèque que Dom João V (1706-1750) érige en symbole de la puissance impériale, à l'heure où l'or de Minas Gerais fait la richesse de Lisbonne et de sa cour. Or, le tremblement de terre de 1755 détruit la quasi-totalité de cette prestigieuse collection. La reconstitution d'un fond passe alors par l'acquisition de collections privées. En 1808, cette Bibliothèque royale gagne Rio de Janeiro afin d'échapper à l'emprise des troupes napoléoniennes. En 1825, lors de la négociation du traité d'Amitié et d'Alliance entre le Portugal et le Brésil, le nouvel Empire du Brésil accepte de payer le prix lourd pour conserver " la meilleure et la plus grande bibliothèque des Amériques : un trophée pour la nouvelle nation et une garantie d'assurance pour le monarque »(p.41). Plus largement, et en étudiant les archives des organes de la censure entre 1769 et 1821, Luiz Carlos Villalta a pu faire état de la circulation croisée des imprimés entre le royaume du Portugal et l'Amérique portugaise. Malgré les nombreuses réformes de la censure, l'État colonial semble agir de façon pérenne en vue de " la défense, en dernier recours, de l'unité de la nation portugaise ", dont la figure $\mathrm{du}$ roi et la religion catholique sont les deux attributs principaux (p. 81). Villalta s'intéresse en particulier aux Livros de Santa Bárbara, livres d'oraison dont plus de 100 000 exemplaires sont débarqués à Rio de Janeiro entre 1795 et 1799. De tels chiffres permettent de relativiser le cliché désormais daté d'une Amérique portugaise analphabète, coupée de tout contact avec les savoirs véhiculés par l'imprimé. À cet égard, Eduardo França Paiva témoigne de l'essor des pratiques de la lecture au sein des populations serviles ou affranchies du Minas Gerais, au XVIII ${ }^{\mathrm{e}}$ siècle. Malgré la censure, de nombreux ouvrages interdits faisaient l'objet d'un commerce de contrebande dynamique et profitable dans l'espace atlantique. L'étude de la bibliothèque du naturaliste José Vieira Couto par Júnia Ferreira Furtado témoigne de la capacité de ces élites brésiliennes formées à l'université de Coimbra à faire fi des interdits en vigueur. Sa bibliothèque, riche de 601 volumes, témoigne de la circulation des idées des Lumières en Amérique portugaise et éclaire la participation de Couto à la Conjuration de Minas Gerais (1789). La carrière de Couto entre Lisbonne et le Brésil reflète également la présence des lettrés luso-brésiliens dans les premiers cercles du pouvoir au début du XIX siècle, notamment dans l'entourage du ministre Dom Rodrigo de Sousa Coutinho. Caio César Boschi traite dans son article de la présence des « Lusobrésiliens » dans les ateliers d'Arco do Cego à Lisbonne, un cercle littéraire et une maison d'édition dont la brève existence incarne alors « la mentalité et les pratiques de gouvernance du réformisme éclairé luso-brésilien » (p. 385).

3 Une autre ligne de force réside dans l'attention accordée aux liens entre l'imprimé politique et le processus de constitution d'une première opinion publique au Brésil. La naissance de la presse au Brésil est contemporaine de l'arrivée de la cour des Bragance à Rio de Janeiro, en 1808. Toutefois, c'est depuis Londres que le premier titre " brésilien » apparaît, à l'initiative d'Hipólito da Costa. Ce dernier s'est formé à Coimbra avant d'être envoyé en mission aux États-Unis pour le compte du royaume. C'est là qu'il est initié à la franc-maçonnerie, qu'il promeut à son retour au Portugal, en 1800, malgré 
l'interdit. Emprisonné, il s'enfuit à Londres où il fonde, comme nous l'explique Isabel Lustosa, le Correio Braziliense (1808-1822). Ce mensuel tiré à moins de 500 exemplaires se destinait d'abord à ses abonnés au Brésil, où il circulait sous le manteau afin d'échapper à la censure. En effet, Costa n'a de cesse d'y dénoncer la mauvaise gestion du royaume, l'institution servile et les monopoles commerciaux. Il soutient l'œuvre des Libertadores sud-américains, à l'instar de Simon Bolivar, tout en vantant pour le Brésil les vertus d'un régime monarchique libéral et éclairé. Cette publication se fait aussi le relais de la légende noire de l'empereur Napoléon Bonaparte : Lúcia Maria Bastos P. Neves étudie la profonde empreinte laissée par cette légende noire dans les sociétés portugaise et brésilienne du début du XIX ${ }^{e}$ siècle. Celle-ci reflète la persistance de croyances de type eschatologique et de "croyances traditionnelles du monde de l'Ancien Régime " (p. 470), avant que n'émerge une première sphère publique politique, à compter des années 1820. C'est ce dont rend compte Marcello Basile, en étudiant la presse de Rio de Janeiro pendant la Régence (1831-1840). Cette période marquée par une forte instabilité politique est aussi un " grand laboratoire politique et social dans lequel des formules diverses et originales furent élaborées et différentes expériences testées, couvrant de larges strates sociales»(p.492). La presse politique contribue alors, malgré la virulence des débats, à promouvoir dans l'opinion publique la nécessité d'œuvrer à la consolidation de la nation brésilienne et de son État.

Enfin, le troisième mérite de cet ouvrage est d'offrir quelques brillantes réflexions quant au rôle déterminant de la circulation atlantique de l'imprimé, et de l'image en particulier, dans les processus de construction nationale en Amérique au XIX ${ }^{\mathrm{e}}$ siècle. Laura Suárez de la Torre illustre à partir du cas mexicain le fait que la construction de représentations nationales s'inscrit dans une circulation internationale des idées et des pratiques culturelles. Verónica Zárate Toscano, quant à elle, explore plus particulièrement la question de la circulation des images à Mexico pour aborder la question de la construction de l'identité nationale. Márcia Abreu nous propose une étude comparée des représentations iconographiques des "Brésiliens " par des artistes étrangers et nationaux au début du XIX ${ }^{\mathrm{e}}$ siècle. Alors que les premiers semblent prompts à mettre en lumière l'exotisme des paysages et le métissage de la société brésilienne, ces derniers préfèrent souligner la dimension civilisée, européenne des élites lettrées impériales. C'est ce dont témoigne également l'étude de Celeste Zenha consacrée à un album de lithogravures, Rio de Janeiro pitoresco (1845), dans la mesure où cet album érige la capitale en symbole de la civilisation impériale, au prix de l'invisibilisation de la présence africaine. C'est à cet idéal que répond aussi, pour une part, la publication du premier Atlas do Império do Brazil (1868), destiné aux élèves du collège impérial Pedro II, étudié ici par Maria Eliza Linhares Borges. Enfin, Antônio Augusto Gomes Batista consacre son étude aux usages des livres scolaires portugais au Brésil ; livres dont la présence permet de défendre la "norme linguistique lusitanienne ", alors que la question de la nationalisation de la langue agite une partie des élites impériales. 